\title{
Nutritional Phases in Prader-Willi Syndrome: Evolutionary and Clinical Interpretations
}

\section{Citation}

Kotler, Jennifer, Karen Balko, Glenn Berall, and David Haig. 2016. "Nutritional Phases in PraderWilli Syndrome: Evolutionary and Clinical Interpretations." Journal of Evolutionary Medicine 4: 1-7. doi:10.4303/jem/235968.

\section{Published Version}

doi: $10.4303 / \mathrm{jem} / 235968$

\section{Permanent link}

http://nrs.harvard.edu/urn-3:HUL.InstRepos:27531388

\section{Terms of Use}

This article was downloaded from Harvard University's DASH repository, and is made available under the terms and conditions applicable to Open Access Policy Articles, as set forth at http:// nrs.harvard.edu/urn-3:HUL.InstRepos:dash.current.terms-of-use\#OAP

\section{Share Your Story}

The Harvard community has made this article openly available.

Please share how this access benefits you. Submit a story.

Accessibility 
Nutritional phases in Prader-Willi Syndrome: evolutionary and clinical interpretations

\author{
Jennifer Kotler ${ }^{1}$, Karen Balko², Glenn Berall ${ }^{2}$ and David Haig ${ }^{1 \mathrm{a}}$ \\ ${ }^{1}$ Department of Organismic and Evolutionary Biology, \\ Harvard University, 26 Oxford Street, \\ Cambridge MA 02138. \\ ${ }^{2}$ Infant Child \& Adolescent Nutrition Clinic \\ 1100 Sheppard Ave. E, Suite 301 \\ Toronto, ON M2K 2W1.
}

Corresponding author: Jennifer Kotler, Department of Organismic and Evolutionary Biology, Harvard University, 26 Oxford Street, Cambridge, MA 02138, USA. E-mail: jkotler@fas.harvard.edu, Tel: $1-617-495-9023$

David Haig, E-mail: dhaig@oeb.harvard.edu

Karen Balko, E-mail: karenannebalko@gmail.com

Glenn Berall, E-mail: glenn.berall@nygh.on.ca

Keywords: Prader-Willi Syndrome; Hyperphagia; Genomic Imprinting; Child development; Appetite; Adrenarche

Page heading title: Nutritional phases in Prader-Willi syndrome.

Word count:

(i) Abstract -1125 characters \& spaces

(ii) $\quad$ Text $-3,977$

(iii) Numbers of tables/figures - 1 


\section{ABSTRACT}

Prader-Willi syndrome (PWS) is caused by a lack of expression of paternally-expressed imprinted genes at human chromosome 15q11-13 and is characterized by a switch from infant anorexia to childhood hyperphagia. A recent multiphase staging system recognizes gradual changes between the anorexic and hyperphagic phases of PWS. We undertook to use clinical records from an independent population to assess the multiphase system and explore the implications for the evolution of distinctive features of human childhood. Medical records of 258 clinic visits by 55 patients with PWS were reviewed with a focus on appetite and feeding. These clinical records were found to be inadequate for placing patients into particular stages of the multiphase system. Under the multiphase system, the onset of hyperphagia in PWS appears to coincide more with the timing of adrenarche than weaning from the breast and this timing should frame future evolutionary hypotheses. We discuss challenges encountered while attempting to use clinical data to explore evolutionary questions, but also identify useful information contained in the records. 


\section{INTRODUCTION}

Humans are one of the most genetically well-studied species, but major gaps remain in scientific knowledge because of ethical and practical constraints on human experimentation. Gene functions in other 'model organisms' can be probed by manipulations that will never, and should never, be performed on human subjects. As a partial substitute, DNA copying errors ('mutations') inevitably occur within the human population and come to medical attention when they affect the health of affected individuals. Thus, medical case records contain extensive replication of rare genetic events that are 'natural experiments' providing clues about gene function. These records could, in principle, allow genetic functions in humans to be probed in ways analogous to 'knockout' experiments in other species, but subject to important constraints that such research be clinically justified and do no harm. An aspiration for evolutionary medicine is to use data from clinical genetics to provide insights into human evolution and then to use these evolutionarily-informed insights to improve clinical practice.

Each patient is unique with individualized medical treatment shaped by their particular symptoms and the idiosyncratic decisions of parents and physicians. Although case studies of rare diseases can be considered natural experiments, medical records often resemble anecdotal natural history more than records in an experimental notebook. One challenge is whether useable information relevant to evolutionary questions can be extracted from data collected for very different purposes. We undertook to explore this question using the medical records of a group of patients with Prader-Willi syndrome (PWS), a rare disorder, with a frequency of between one in 10,000 and 30,000 livebirths [1].

In 1989, cytologically indistinguishable deletions of human chromosome $15 q$ were shown to be associated with a diagnosis of PWS when the deletion occurred on the chromosome inherited from a child's father but Angelman syndrome (AS) when the deletion occurred on the chromosome inherited from the child's mother. Therefore, PWS and AS were strongly suspected of being caused by disordered expression of genes that were differentially expressed when inherited via eggs or sperm [2]. 
In the same year, genomic imprinting was proposed to have evolved in response to intragenomic conflict between genes of maternal and paternal origin [3]. Since then the role of imprinted genes in the etiology of PWS and AS has been confirmed [4] and the phenotypes of these genetically-paired syndromes have been interpreted as providing clues about the role of kin interactions in the evolution of distinctive features of human childhood [5-8].

PWS particularly attracted attention of evolutionary biologists because its primary symptoms involve perturbations of childhood appetite, suggesting that kin-mediated conflicts have shaped the evolution of childhood feeding behaviors and that the phenotype of PWS may provide clues about the roles of matrilineal and patrilineal kin in caring for ancestral children at different stages of their development. PWS was traditionally characterized as having two nutritional phases: an early phase in which infants fed poorly and were disinterested in food; and a later phase in which children became hyperphagic, obsessed with food, and morbidly obese [9]. The age at which children with PWS shifted from 'anorexia' to 'hyperphagia' was suggested to begin in the second or third year of postnatal life [5,10]. Previous attempts to interpret changing appetite in PWS from an evolutionary perspective have been based on this two-phase model. The early 'anorexic' phase was readily explicable in terms of maternal-paternal conflict over infant care $[5,8]$. Paternally-expressed genes (PEGs) from the PWS/AS chromosomal region were proposed to have evolved to increase nutritional demands on mothers by promoting more intense suckling and frequent waking. In the absence of expression of these PEGs, infants with PWS exhibit weak suck and prolonged sleep.

The later 'hyperphagic’ phase posed a more formidable puzzle for evolutionary interpretation. How could increased appetite in the absence of expression of PEGs be reconciled with the theoretical prediction that PEGs increase costs to mothers or their kin? The anthropological literature suggests humans typically introduced supplemental foods at about six months, with cessation of breast-feeding in the third year [11]. If the onset of hyperphagia is in the second or third year, as envisaged in the twophase model of PWS, appetite increased at roughly an age at which our ancestors were being weaned 
from the breast. Therefore, Haig and Wharton proposed that PEGs from the PWS region had been selected to delay weaning by causing infants to resist substitution of alternative foods for breast milk [5]. As an alternative, Úbeda proposed that weaning was evolutionarily associated with a shift from reliance of children on mothers for nutrition to greater dependence on fathers and their kin [6]. A choice between these models requires a better understanding of typical patterns of provisioning in the evolutionary past and a better understanding of the phenotype of PWS.

A recent synthesis of the clinical experience of eminent researchers in the field has replaced the two-phase model of nutritional development in PWS with a more complex model of seven phases and sub-phases [10]. These are (0) decreased fetal movements; (1a) hypotonia, feeding difficulty and decreased appetite (0-9 months); (1b) improved feeding and appetite with appropriate growth (9-25 months); (2a) increasing weight without enhanced appetite or excessive caloric intake (2.1-4.5 years); (2b) increased appetite and caloric intake without hyperphagia (4.5-8 yrs); (3) hyperphagia (after 8 years); (4) satiable appetite (present in two adults). From an evolutionary perspective, the initial improvement of appetite in the new multiphase model coincides roughly with the ancestral period of human weaning, but the onset of frank hyperphagia is too late to be explicable in these terms.

We undertook to assess the new model in a retrospective review of medical records at one of the largest PWS clinics in Canada. The control of food intake is a major clinical concern for patients with PWS and their caregivers, and our long-term hope is that a better understanding of the evolutionary forces that have shaped developmental changes in human appetite may provide insights into more effective treatment of excessive eating in PWS.

\section{METHODS}

A total of 258 retrospective chart reviews were conducted of 55 patients with PWS (26 female, 29 male) at the Infant Child \& Adolescent Nutrition Clinic (ICAN) in North York General Hospital 
(Toronto). Patients varied in age from 6 weeks to 51 years (mean $=11.6$ years), with $1-34$ clinic visits per patient (median =6). All data collected in these chart reviews were based on the standardized PWS collection sheets used at ICAN, as well as a thorough review of any external testing, reports from specialists, and anecdotal reports from family or community staff recorded in each patient's file.

Genetic testing was conducted by local hospital facilities under the referral of the families’ physicians, as per standard clinical practice. All patients had a phenotypic diagnosis of PWS, but only 33 (60\%) had undergone genetic testing. Of these patients, a genetic cause of PWS was identified in 27 cases ( $n=4$ maternal uniparental disomy, $n=23$ deletion), but no genetic cause could be determined in six cases (18\% of those tested). Most patients (14/21) whose first visit occurred before 5 years old had a molecular diagnosis, while only 9/28 patients whose first visit occurred after 10 years old had a molecular diagnosis. The difference in rates of genetic testing by age is not surprising; genetic tests for PWS are usually conducted soon after birth and were not standard practice, or had not yet been developed, when older patients were infants.

The standard treatment recommended to parents at ICAN is not to introduce supplemental foods until 10 months of age because of hypotonia, which affects feeding. Upon introduction of supplemental foods, a sheet is used that exchanges breastmilk or formula for an equal calorie amount of purees to prevent excess weight gain. Parents are instructed in the red-yellow-green system of food choices [12].

\section{RESULTS}

\section{APPLICATION OF THE NEW STAGING SYSTEM}

Our primary finding was that the chart reviews contained inadequate information to assign individuals with PWS to one of the sub-phases described by Miller and colleagues. Our analysis also identified some general difficulties with application of this staging system to clinical populations. The new scheme is based on the impressions of authors with long clinical experience of working with children 
with PWS and includes some subjective elements. For example, the transition from decreased appetite (1a) to improved appetite (1b, 2a) to enhanced appetite (2b) to hyperphagia (3) is a continuum without explicit criteria of where to draw lines between stages. Moreover, some criteria used in staging are not routinely included in medical records. For example, clinics usually do not record body fat measures for children under the age of nine, by which point most children with PWS would already be in phase (3). Even were better data available, there is no clear guidance how clinicians should apply it to sort their patients into nutritional phases. None of the statistical analyses provided by Miller and colleagues demonstrate a linear progression of stages, nor do their analyses control for changes with age. The categorization of stages is therefore somewhat arbitrary and creates ambiguity when placing patients in a particular stage.

While the primary symptoms of PWS are related to feeding and growth, individuals with PWS also exhibit characteristic behavioral disturbances, including temper tantrums, obsessions, compulsions, sudden acts of violence, and poor social behavior. Such behavioral outbursts are often related to food restrictions but can occur without obvious provocation [13], and can cause familial and social disruptions. While behavioral interventions can be effective [14], psychiatric treatment is often implemented, including medication. Most patients in our study aged 15 years or older were prescribed at least one psychiatric medication (45/83 visits), with most taking combinations of drugs, sometimes twenty or more. Many psychiatric drugs are known to suppress or enhance appetite. Without controlling for effects of medication while evaluating the progression of PWS, physicians may misinterpret drug-induced changes of appetite as ontogenetic stages of the disease. This is a particular concern for the later stages of the multiphase system, which relies on subtle changes in appetite for patients in this age category. Almost half of our patients $(n=20)$ had been prescribed Topirimate as adults (age 22-33 years) specifically for its appetite suppressing effects. In fact, 28/55 patients had been on at least one appetite modifying (suppressing or enhancing) drug; the heavy use of prescription medication in this population creates uncertainty about whether observed changes in appetite in adults 
are due to medication or are part of the unmedicated natural history of PWS.

Other variations in treatment could also influence assignment of nutritional phases. For example, twenty patients in our study spent their adolescence and adult years in group homes where food intake was carefully controlled, including keeping food locked and out of sight, and working with a dietician to implement predictable meal plans and schedules [15]. This has been shown to help patients feel satisfied, allow them to shift their focus from food-oriented activities, and help to control many behavioral problems [14]. Because of the way these living situations are designed, later nutritional phases (specifically phase 4 - an ability to feel full) might measure the efficacy of treatment, not the natural progression of PWS. All of the 13 patients who reported the ability to feel satiated during at least one clinic visit lived in food-secure environments (family home or group home).

The only patient in our sample who was identified as living independently required significant supervision and dietary control in order to maintain a healthy weight. This patient had not 'achieved' phase 4 at the age of 49 yrs. Only two of 82 patients in Miller and colleagues' population were considered to have entered the fourth and final stage. Thus the evidence for an adult satiety stage in the natural progression of PWS is weak.

\section{OTHER CLINICAL FINDINGS}

Our chart reviews provided a variety of evidence on feeding behaviors during the progression of PWS. Of the eleven patients for whom we have feeding information from the first six months of postnatal life (34 separate visits), only three mothers attempted to breast feed. Two of these attempted to breast feed before their infants were two months of age, while the third did not begin breast feeding until her six month visit. These mothers were dedicated to making breast feeding work, but none was fully successful (see Table 1 for a summary of infant feeding histories of these patients). Based on their medical records, only seven of the 55 individuals with PWS in our study had a history of breastfeeding. 
Of this small group, three required nasogastric intubation at birth, and all required supplementation by infant formula and/or expressed breast milk during the breastfeeding period. A total of 24 infants were given expressed breast milk (usually in combination with infant formula). Of these infants, seven were not only unable to breastfeed, but also required assistance in bottle-feeding (e.g. Haberman bottle, compressible bottle, syringe feeding, enlarged nipple hole).

We were unable to assess the age of transition to solid foods, although many parents anecdotally commented that this transition was an easy one for their child with PWS. One mother with twin boys noted that her son with PWS showed much more interest in solid foods than his twin brother without PWS, while still showing the typical PWS aversion to fluids (absent in his brother without PWS).

Behaviors associated with the introduction of solid foods would be an area of clinical and evolutionary interest for further exploration.

Individuals with PWS are often described as having a non-discriminating appetite, but 17 of our 55 patients (9 deletion cases, 1 maternal uniparental disomy, 7 unknown or not tested) were described by parents or care workers as ‘picky eaters’ over 49 separate clinic visits. These individuals ranged in age from 11 months to 32 years. Interestingly, one of our patients was described by his mother as significantly more fastidious than his twin brother without PWS, showing aversions to proteins and some fruits (34 months old). Food preferences showed clear patterns with age: all picky eating between 11 to 29 months involved texture sensitivity (refusal to eat meat or non-pureed/chunky foods). One possibility is that these patients had developed oral aversions secondary to prolonged gastric feeding. An aversion to meat and dairy (including milk) was quite common throughout the age groups, as well as an increased preference for starchy foods.

\section{DISCUSSION}

Miller and colleagues present a more nuanced model of nutritional phases in PWS. Specifically, their 
system of disease progression includes an ontogenetic separation of slow metabolism (weight increase, phase 2a) from increased interest in food (hyperphagia, phase 2b). This gradual shift in feeding behaviors has been noted to some extent in previous research, which found that weight gain began at around age 4-5 months, and normalized between the ages of 15-30 months. Furthermore, it was noted that parental reports of increased interest in food and eating behaviours beyond normal occurred after the age at which BMI started to increase beyond the normal range [16]. However, anecdotal reports from parents indicated eating behaviors that differed qualitatively from a young age, leading the authors to consider the possibility that hypotonia during infancy as well as timely implementation of strict feeding controls may have masked early signs of hyperphagia in this group. They found that any relaxation of feeding controls, even at a young age, resulted in increases in BMI [16].

Previous evolutionary interpretations of the phenotype of PWS have been based on the twophase model of disease progression in which children with PWS change from disinterest in food to hyperphagia at some time during the first three postnatal years. Haig and Wharton suggested that PEGs from the PWS region had evolved to promote suckling during early infancy and to resist introduction of alternative foods at the time of weaning [5]. The major benefit for infants was conjectured to have been longer delays until births of younger sibs caused by prolongation of their mothers' lactational amenorrhea [8]. Conception is followed by birth nine months later. The benefit for the suckling child of delaying the conception of a younger sib would have been greatest during the first year but would have rapidly declined with postnatal age. Furthermore, this non-nutritional benefit of night waking and suckling would disappear once the mother was pregnant with a younger sib (new pregnancy is a common reason for weaning in non-contracepting populations $[17,18])$.

The transition from poor appetite (stage 1a) to improved appetite (stage 1b) occurs at about nine months of age in the multiphase model of Miller and colleagues. This early gain in appetite is compatible with the model of Haig and Wharton that relates increase of appetite to the ancestral age of weaning [1]. However, hyperphagia and associated behaviors do not become prominent until about 
eight years (stage 3) and appear unlikely to be explicable in terms of evolutionary conflicts associated with weaning from the breast to solid foods.

The onset of hyperphagia in PWS roughly coincides with middle childhood and adrenarche [19-21]. Adrenarche occurs at around the age of weaning in great apes: bonobos wean after four years [22] with increases in adrenal androgens after five years [23]; orangutans wean after seven years [24] with increases in adrenal androgens after eight years [25]. Campbell noted that increased production of adrenal androgens accompanies independence from mothers at weaning in rodents and monkeys but occurs much later than weaning in humans [26]. Early hominins were probably weaned at adrenarche as occurs in other great apes. The origin of 'complementary feeding' can be conjectured to have allowed human mothers to conceive another child before its older sib was nutritionally independent [7]. Thus, human weaning can be considered to occur in two stages: from breast milk to a special infant diet and from this diet to adult food. In this interpretation, the 'five to seven year shift' of middle childhood in modern humans coincides with the second stage of weaning and is behaviorally homologous to the increased independence that occurs at weaning from the breast in other great apes.

Adrenarche occurs early in PWS [27] implying that PEGs from the PWS chromosomal region directly or indirectly inhibit production of adrenal androgens. We conjecture that behavioral changes associated with adrenarche reduced the demands of our ancestors on their mothers' time and attention, possibly including time spent by mothers in collecting and preparing special foods. As a consequence maternally-derived genes expressed in infants evolved to favor earlier adrenarche than paternallyderived genes. It would be interesting to know whether changes in appetite in PWS are correlated with changes in the production of adrenal androgens.

Food-seeking behaviors in PWS have been variously described as 'foraging' or ‘food-stealing' [28] but the alternative labels have very different connotations in everyday life and suggest different evolutionary interpretations. 'Foraging' suggests a prosocial behavior that benefits kin by reducing the costs of provisioning the child, whereas 'food stealing' suggests an antisocial behavior in which the 
child takes food from other group members [7]. Clarification of symptomatic differences between these two descriptors might help further evolutionary understanding. Observations that some individuals with AS develop hyperphagia [29-32] and that a few males with PWS develop anorexia nervosa [33,34] add further complications. Clearly, there is much still to be learned about the natural history of appetite and food-related behaviors in PWS and AS.

\section{FUTURE DIRECTIONS}

Humans wean offspring sooner than their closest nonhuman relatives and consequently have shorter interbirth intervals. As a consequence, care of successive offspring overlaps more than in other great apes, made possible by social support of mothers and special post-weaning child diets. Weaning is followed by a prolonged period of slow growth before our distinctive pubertal growth spurt [35]. We believe that the greatest contribution of evolutionary thinking to understanding phenotypic changes in PWS will come from viewing these changes within a conceptual framework of increasing independence from maternal care during childhood and associated changes in feeding.

The phenotype of infants with PWS fits evolutionary models in which PEGs have been selected to enhance suckling and nightwaking because of a benefit to infant fitness at a cost to maternal fitness [5-8]. While our paper has focused on feeding behaviours, the phenotype of older children with PWS promises to provide similar insight into kin-mediated tradeoffs in the evolution of other distinctive aspects of human childhood. For example, adrenarche occurs early in children with PWS [27] but puberty is generally considered to be delayed and incomplete [36,37]. Recently, mutations in the paternally-derived copy of MKRN3, a PEG from the PWS region, have been identified as a major cause of precocious puberty [38], but early puberty is not a diagnostic feature of PWS despite the absence of expression of MKRN3. This suggests that other imprinted genes from the PWS region interact with $M K R N 3$ in the timing and progression of sexual maturation. An improved understanding of the dissociation of various aspects of adrenarcheal and pubertal progression in PWS may provide insight 
into the complex selective forces acting on the age of sexual maturation during human evolution. Going forward, evolutionary theory may also be applied to other relevant PWS symptoms, including psychological perturbations, in order to more fully understand the role of genetic conflict in human evolution.

\section{CONCLUSION}

Miller and colleagues’ new system raises important questions for both evolutionary research and clinical practice, but we encountered difficulties in applying the new system to a different patient population. We found that treatment methods, including both behavioral strategies and psychiatric medications, are important variables that need to be considered in describing the 'natural' progression of PWS. Furthermore, standard clinical collection tools often do not include the information required to place individuals in the multiphase system, rendering it difficult to implement in the context of current clinical practice.

Finally, we found little evidence for the final 'satiable appetite' phase, although GB notes that hyperphagia, in his clinical experience, may be less intense in older patients. This might be due, in part, to many years of behavioral modification training, but further research into this area is warranted. The inclusion of this final stage of satiety could illicit anxiety and negative self-perception in both patients and families of patients who fail to attain feelings of satiety. We therefore consider inclusion of a satiety phase to be clinically premature before further research shows that its achievement can be considered a realistic goal for adults with PWS.

From a methodological perspective, our pilot study identifies limitations of existing clinical data for the evolutionarily-informed study of rare human diseases whose phenotypes develop over many years. Because of the rarity of PWS, any particular clinic will have a relatively small heterogenous group of patients of mixed ages. All will receive treatments that differ depending on parental and 
physician choices, and control groups of untreated patients will be unavailable for obvious ethical reasons. Moreover, accepted standards of care will change over time so that cross-sectional studies confound age-differences with treatment differences.

Despite these caveats, clinical data do contain useable information about appetite in PWS. The new multiphase system is likely to improve the quality of this information because of the systems’ attention to subtle and gradual changes in appetite. Moreover, evolutionary analysis can suggest kinds of information that would be simple to collect and that would help better understand the natural history of PWS. We are particularly interested in a possible relation between markers of adrenarche and changes in appetite and behavior.

While some evidence supports the multiphase model of nutritional phases in PWS, more work needs to be done before this system can be applied to clinical settings or evolutionary questions. Future studies should amend the statistical analyses to better adjust for age and to demonstrate linear phase progression. Moreover, it is important to control for treatment methodology, including both medication and behavioral interventions. Finally, clearer guidelines should be developed to assure correct implementation of the new staging system by PWS clinics.

\section{ACKNOWLEDGEMENTS}

The authors thank the Foundation for Prader-Willi Research for funding this work.

The authors declare that there are no conflicts of interest. 
1. Cassidy SB, Schwartz S, Miller JL et al. Prader-Willi syndrome. Genet Med 2012;14:10-26.

2. Knoll JH, Nicholls RD, Magenis RE et al. Angelman and Prader-Willi syndromes share a common chromosome 15 deletion but differ in parental origin of the deletion. Am J Med Genet 1989;32:285-90.

3. Haig D, Westoby M. Parent-specific gene expression and the triploid endosperm. Am Nat 1989;134:147-55.

4. Nicholls RD, Knepper JL. Genome organization, function, and imprinting in Prader-Willi and Angelman syndromes. Annu Rev Genomics Hum Genet 2001;2:153-75.

5. Haig D, Wharton R. Prader-Willi syndrome and the evolution of human childhood. Am J Hum Biol 2003;15:320-9.

6. Úbeda F. Evolution of genomic imprinting with biparental care: implications for Prader-Willi and Angelman syndromes. PLoS Biol 2008;6:1678-92.

7. Haig D. Transfers and transitions: parent-offspring conflict, genomic imprinting, and the evolution of human life history. Proc Natl Acad Sci U S A 2010;107:1731-5.

8. Haig D. Interbirth intervals : and intrasomatic conflict. Evol Med Public Heal 2014:1-18.

9. McAllister CJ, Whittington JE, Holland AJ. Development of the eating behaviour in PraderWilli Syndrome: advances in our understanding. Int J Obes (Lond) 2011;35:188-97.

10. Miller JL, Lynn CH, Driscoll DC et al. Nutritional phases in Prader-Willi syndrome. Am J Med Genet A 2011;155A:1040-9.

11. Sellen DW, Smay DB. Relationship between subsistence and age at weaning in “preindustrial” societies. Hum Nat 2001;12:47-87.

12. Balko K. Red Yellow Green System for Weight Management. Toronto, ON: Ontario PraderWilli Syndrome Association, 2005.

13. Butler, Merlin G., Lee, Phillip D. K., Whitman, Barbara Y. ed. Management of Prader-Willi Syndrome. 3rd ed. New York: Springer, 2006.

14. Dykens E, Shah B. Psychiatric disorders in Prader-Willi syndrome: epidemiology and management. CNS Drugs 2003;17:167-78.

15. Hoffman CJ, Aultman D, Pipes P. A nutrition survey of and recommendations for individuals with Prader-Willi syndrome who live in group homes. J Am Diet Assoc 1992;92:823-30, 833.

16. Butler J V, Whittington JE, Holland AJ et al. The transition between the phenotypes of Prader-Willi syndrome during infancy and early childhood. Dev Med Child Neurol 2010;52:e88-93.

17. Bohler E, Bergström S. Premature weaning in East Bhutan: only if mother is pregnant again. J Biosoc Sci 2008;27:253-65.

18. Valeggia C, Ellison PT. Lactational Amenorrhoea in Well-Nourished Toba Women of Formosa, Argentina. J Biosoc Sci 2004;36:573-95.

19. Lancy DF, Grove MA. Getting noticed. Middle childhood in cross-cultural perspective. Hum Nat 2011;22:281-302.

20. Del Giudice M. Middle Childhood: An Evolutionary-Developmental Synthesis. Child Dev 
Perspect 2014;8:193-200.

21. Campbell B. Adrenarche and the evolution of human life history. Am J Hum Biol 2006;18:569-89.

22. De Lathouwers M, Van Elsacker L. Comparing infant and juvenile behavior in bonobos (Pan paniscus) and chimpanzees (Pan troglodytes): a preliminary study. Primates 2006;47:287-93.

23. Behringer V, Hohmann G, Stevens JMG et al. Adrenarche in bonobos (Pan paniscus): evidence from ontogenetic changes in urinary dehydroepiandrosterone-sulfate levels. J Endocrinol 2012;214:55-65.

24. van Noordwijk MA, van Schaik CP. Development of ecological competence in Sumatran orangutans. Am J Phys Anthropol 2005;127:79-94.

25. Prall SP, Ambu L, Nathan S et al. Androgens and innate immunity in rehabilitated semicaptive orangutans (Pongo pygmaeus morio) from Malaysian Borneo. Am J Primatol 2015;77:642-50.

26. Campbell B. Adrenarche in comparative perspective. Am J Hum Biol 23:44-52.

27. Siemensma EPC, de Lind van Wijngaarden RF a, Otten BJ et al. Pubarche and Serum Dehydroepiandrosterone Sulfate Levels in Children with Prader-Willi Syndrome. Clin Endocrinol (Oxf) 2011;31:83-9.

28. Page TJ, Finney JW, Parrish JM et al. Assessment and reduction of food stealing in praderwilli children. Appl Res Ment Retard 1983;4:219-28.

29. Fridman C, Varela MC, Nicholls RD et al. Unusual clinical features in an Angelman syndrome patient with uniparental disomy due to a translocation 15q15q. Clin Genet 1998;54:303-8.

30. Fridman C, Varela MC, Valente K et al. Phenotypic and behavioral variability within Angelman Syndrome group with UPD. Genet Mol Biol 2002;25:127-30.

31. Poyatos D. Severe phenotype in Angelman syndrome resulting from paternal isochromosome 15. $J$ Med Genet 2002;39:4e - 4 .

32. Freeman SB, May KM, Pettay D et al. Paternal uniparental disomy in a child with a balanced 15;15 translocation and Angelman syndrome. Am J Med Genet 1993;45:625-30.

33. Counts D. An adult with Prader-Willi syndrome and anorexia nervosa: A case report. Int $J$ Eat Disord 2001;30:231-3.

34. Whittington J, Holland T. Prader-Willi Syndrome: Development and Manifestations. Cambridge: Cambridge University Press, 2004.

35. Sellen DW. Evolution of infant and young child feeding: implications for contemporary public health. Annu Rev Nutr 2007;27:123-48.

36. Siemensma EPC, van Alfen-van der Velden AAEMJ, Otten BJ et al. Ovarian function and reproductive hormone levels in girls with Prader-Willi syndrome: a longitudinal study. J Clin Endocrinol Metab 2012;97:E1766-73.

37. Siemensma EPC, de Lind van Wijngaarden RFA, Otten BJ et al. Testicular failure in boys with Prader-Willi syndrome: longitudinal studies of reproductive hormones. J Clin Endocrinol Metab 2012;97:E452-9.

38. Abreu AP, Dauber A, Macedo DB et al. Central precocious puberty caused by mutations in the imprinted gene MKRN3. N Engl J Med 2013;368:2467-75. 
Table 1: Feeding patterns for 11 patients (5 F) over 34 visits during the first 6 months of development. $(E B M=$ expressed breast milk)

\begin{tabular}{|c|c|c|c|c|c|c|}
\hline Gender & Genetic Subtype & Age (months) & NG Tube & Breast & EBM & Formula \\
\hline \multirow{4}{*}{ Male } & \multirow{4}{*}{ mUPD } & 3.5 & $\checkmark$ & & & \\
\hline & & 5 & $\checkmark$ & & & $\checkmark$ \\
\hline & & 5.33 & $\checkmark$ & & & $\checkmark$ \\
\hline & & 6 & & & & $\checkmark$ \\
\hline \multirow{4}{*}{ Male } & \multirow{4}{*}{ Deletion } & 2 & $\checkmark$ & & $\checkmark$ & $\checkmark$ \\
\hline & & 3 & & & $\checkmark$ & \\
\hline & & 4 & & & $\checkmark$ & \\
\hline & & 5 & & & $\checkmark$ & \\
\hline \multirow{3}{*}{ Female } & \multirow{3}{*}{ Deletion } & 3 & & & $\checkmark$ & $\checkmark$ \\
\hline & & 4 & $\checkmark$ & & $\checkmark$ & $\boldsymbol{V}$ \\
\hline & & 6 & $\checkmark$ & & $\checkmark$ & $\checkmark$ \\
\hline \multirow{4}{*}{ Female } & \multirow{4}{*}{ Deletion } & 2 & $\checkmark$ & $\checkmark$ & $\checkmark$ & $\checkmark$ \\
\hline & & 3 & & & $\checkmark$ & $\checkmark$ \\
\hline & & 4.5 & & & $\checkmark$ & $\checkmark$ \\
\hline & & 6 & & & $\checkmark$ & $\checkmark$ \\
\hline \multirow{4}{*}{ Female } & \multirow{4}{*}{ mUPD } & 1.5 & $\checkmark$ & $\checkmark$ & $\checkmark$ & $\checkmark$ \\
\hline & & 3 & & & $\checkmark$ & $\checkmark$ \\
\hline & & 4 & & & $\checkmark$ & $\checkmark$ \\
\hline & & 5 & & & $\checkmark$ & $\checkmark$ \\
\hline \multirow{2}{*}{ Male } & \multirow{2}{*}{ Not tested } & 4 & $\checkmark$ & & & $\checkmark$ \\
\hline & & 5 & & & & $\checkmark$ \\
\hline \multirow{4}{*}{ Male } & \multirow{4}{*}{ Not tested } & 2 & $\checkmark$ & & & $\checkmark$ \\
\hline & & 4 & $\checkmark$ & & & $\checkmark$ \\
\hline & & 5 & & & & 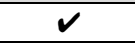 \\
\hline & & 6 & $\checkmark$ & & & $\checkmark$ \\
\hline Female & Deletion & 4 & $\checkmark$ & & $\checkmark$ & $\checkmark$ \\
\hline \multirow{5}{*}{ Female } & \multirow{5}{*}{ Deletion } & 2 & $\checkmark$ & & $\checkmark$ & \\
\hline & & 3 & & & $\checkmark$ & \\
\hline & & 4 & & & 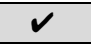 & \\
\hline & & 5 & & & $v$ & \\
\hline & & 6 & & $\checkmark$ & $v$ & \\
\hline Male & mUPD/IC & 4 & $\checkmark$ & & & $\checkmark$ \\
\hline \multirow{2}{*}{ Male } & \multirow{2}{*}{ Deletion } & 4 & $\checkmark$ & & $\checkmark$ & $\checkmark$ \\
\hline & & 5 & & & $\checkmark$ & $\checkmark$ \\
\hline
\end{tabular}

